\title{
Histopathology of the right ventricular outflow tract and its relationship to clinical outcomes and arrhythmias in patients with tetralogy of Fallot
}

Ujjwal K. Chowdhury, MCh, Siddharth Sathia, MCh, Ruma Ray, MD, MRCPath, Rajvir Singh, MSc, PhD, Kizakke K. Pradeep, MCh, and Panangipalli Venugopal, MCh

Supplemental material is available online.
From the Cardiothoracic Centre, All India Institute of Medical Sciences, New Delhi, India.

Received for publication Jan 13, 2006; revisions received March 28, 2006; accepted for publication April 4, 2006.

Address for reprints: Ujjwal K. Chowdhury, MCh, Diplomate NB, Additional Professor, Department of Cardiothoracic and Vascular Surgery, All India Institute of Medical Sciences, New Delhi-110029, India (E-mail: ujjwalchow@rediffmail.com).

J Thorac Cardiovasc Surg 2006;132:270-7

$0022-5223 / \$ 32.00$

Copyright $\odot 2006$ by The American Association for Thoracic Surgery

doi:10.1016/j.jtcvs.2006.04.001
Objectives: The purposes of this study were to evaluate the myocardial histopathology and ultrastructure in patients with tetralogy of Fallot and to identify the histopathologic characteristics that may predispose patients to postoperative myocardial dysfunction and arrhythmias.

Patients and Methods: Operatively resected crista supraventricularis muscle from 183 patients undergoing intracardiac repair of tetralogy of Fallot aged 12 months to 42 years (mean, $106.84 \pm 79.35$ months) were studied by light and electron microscopy. Biventricular function and cardiac rhythm were assessed by 2-dimensional echocardiography and electrocardiography.

Results: The incidence of moderate or severe cellular hypertrophy, endocardial thickening, and interstitial fibrosis was 36\%,68.3\%, and 65\%, respectively. Logistic regression analysis demonstrated age greater than 4 years, systemic arterial desaturation, higher hematocrit values, and elevated ventricular end-diastolic pressures as the major predisposing risk factors for pathologic changes. Twenty-seven $(81.8 \%)$ patients more than 15 years of age and $29(29.3 \%)$ patients between 4 and 15 years of age had predominant right ventricular dysfunction and low cardiac output $\left(\chi^{2}[1\right.$ degree of freedom $(d f)]=27.95 ; P<.001$; odds ratio $[\mathrm{OR}]=10.86$ [3.75-33.10]). Ventricular arrhythmia was detected in 11 patients in whom repair was performed between 4 and 15 years of age and in 13 patients whose age at operation was 15 years or older. According to an additive logistic model, the effect of age at repair on the influence of ventricular arrhythmia was significant $\left(\chi^{2}[1 \mathrm{df}]=24.4 ; P<.001\right.$; $\mathrm{OR}=8.21(2.96-23.11])$.

Conclusions: The great majority of myocardial tissues in cyanotic tetralogy of Fallot indicates pre-existing ultrastructural hypertrophic and degenerative changes. The changes are more pronounced in older patients subjected to long-standing cyanosis and pressure overload and may account for or may coexist with the higher incidence of myocardial dysfunction and ventricular arrhythmia.

$\mathrm{E}$ arly repair of tetralogy of Fallot (TOF) is advocated to minimize organ damage due to chronic hypoxia and to reduce long-standing pressure overload of the right ventricle and arrhythmia. ${ }^{1-3}$ The time point when chronic hypoxia leads to irreversible ventricular dysfunction is unknown. Older patients with TOF have a higher postoperative inotropic requirement, and its effect on late survival will mandate continued investigation. ${ }^{2}$ Whether this is related to downregulation of receptors on the myocardial cells or to progressive alteration in myocyte architecture remains to be elucidated. Infundibular spasm is one of the causative factors for cyanotic spells, which are maximal in infancy, respond to propranolol administration, and decrease with age owing to progressive replacement of the infundibular 


$$
\begin{aligned}
& \text { Abbreviations and Acronyms } \\
& \begin{aligned}
\mathrm{CI} & =\text { confidence interval } \\
d f & =\text { degree of freedom } \\
\mathrm{OR} & =\text { odds ratio } \\
\mathrm{RVEDP} & =\text { right ventricular end-diastolic pressure } \\
\mathrm{RVOT} & =\text { right ventricular outflow tract } \\
\mathrm{TOF} & =\text { tetralogy of Fallot }
\end{aligned}
\end{aligned}
$$

muscle with fibrous tissue producing fixed obstruction. ${ }^{1-3}$ However, there is no histologic evidence to lend credence to this hypothesis.

This prospective study aims (1) to elucidate the histopathologic characteristics of the resected right ventricular outflow tract (RVOT) muscle in patients with TOF; (2) to correlate the anatomic diagnosis, age at operation, systemic arterial oxygen saturation, and right ventricular end-diastolic pressure (RVEDP) to the morphologic changes; and (3) to identify the morphologic characteristics that may predispose patients to a higher risk of postoperative myocardial dysfunction and arrhythmias.

\section{Patients and Methods}

The study was approved by the Institutional Review Board of the All India Institute of Medical Sciences, and patients were entered in the histopathologic study protocol after informed consent was obtained from parents/guardians. Between January 2002 and June 2005, specimens of resected RVOT muscle from 194 consecutive patients undergoing intracardiac repair of TOF at the All India Institute of Medical Sciences, New Delhi, India, were exposed to histopathologic analysis. Of these, 183 samples from 183 patients (107 boys) were found suitable for histopathologic analysis (11 were excluded owing to morphologic artifacts resulting from inadequate fixation). The examiners were blind to demographic, procedural, and hemodynamic data.

Age at correction was 12 months to 42 years (mean, $106.84 \pm$ 79.35 months; median, 80.0 months), with $27.8 \%$ of patients ( $\mathrm{n}=$ 51 ) being younger than 4 years of age. Cardiac catheterization and angiocardiography were performed on all patients to confirm the diagnosis, to define coronary artery anatomy, and to identify major aortopulmonary collateral arteries. The details of the patients are summarized in Table E1 and Figure E1.

Standard cardiopulmonary bypass and myocardial protection techniques were used in all patients. Intracardiac repair was performed with a transatrial-transpulmonary approach in 147 patients and a trans-right atrial approach in 36 patients. In 145 patients, a transannular patch was used. Surgery/cryoablation for supraventricular or ventricular arrhythmias was not required in any patient. The tissues studied were operatively resected from the parietal band of the crista supraventricularis.

\section{Investigations}

Analysis of cardiac rhythm. Preoperative (baseline) and late postoperative 12-lead electrocardiographic parameters were anal- yzed by a cardiologist who was blinded to the clinical data. Ambulatory electrocardiograms were obtained by 24-hour Holter studies, when the 12-lead electrocardiogram showed arrhythmias, or when patients had symptoms suggestive of arrhythmias. A Mortara H-12 Holter unit (Mortara Instruments Inc, Milwaukee, Wis) was used for monitoring in 78 patients.

The diagnoses of sinus node dysfunction and arrhythmias were made according to standard criteria. ${ }^{4}$ Significant arrhythmia was defined as (1) sustained atrial flutter/fibrillation or sustained monomorphic ventricular tachycardia documented on a 12-lead electrocardiogram or by Holter monitoring or (2) palpitations associated with syncope or near syncope in patients who had subsequently inducible sustained atrial flutter/fibrillation or sustained monomorphic ventricular tachycardia lasting more than 30 seconds or for any length of time if associated with hemodynamic compromise. Ventricular arrhythmias were graded by a modification of the Lown criteria. ${ }^{4}$

Echocardiographic analysis. Echocardiographic studies were performed on all patients before and after the operation, per the American Society of Echocardiography Criteria. ${ }^{5}$ Variables studied included atrioventricular valve regurgitation, status of RVOT, biventricular size and function, and flow through the fenestration. Restrictive physiology was defined from the presence of laminar antegrade diastolic pulmonary arterial flow throughout the respiratory cycle. Pulmonary regurgitation was graded as mild, moderate, or severe.

Collection and preparation of tissues. Resected RVOT muscle during intracardiac repair of TOF was subjected to histopathologic evaluation by light and electron microscopy.

LIGHT MICROSCOPIC EVALUATION. For light microscopy, the specimens were fixed in $10 \%$ buffered formalin and paraffin blocks were prepared. Sections 4 to $5 \mu \mathrm{m}$ thick were cut and were stained by hematoxylin and eosin. Special staining using Masson's trichrome and elastic van Gieson was performed when required, and the sections were examined with a research light microscope (model B-50; Olympus Corp, Tokyo, Japan; magnification $80 \times$ or $160 \times)$. The following parameters were evaluated during light microscopic examination: endocardial thickening, degree and nature of interstitial fibrosis (perivascular, replacement, or interfiber type), and status of the myocyte including myocyte hypertrophy. Each of these parameters was graded as mild, moderate, or severe according to severity.

ELECTRON MICROSCOPIC EVALUATION. For electron microscopic analysis, muscle biopsy tissue obtained at the time of intracardiac repair was immediately minced into 1 - to 2 -mm pieces and fixed in cold buffered $3 \%$ glutaraldehyde solution for 12 hours. Postfixation was carried out for 2 hours with osmium tetroxide. Blocks were prepared and $1-\mu \mathrm{m}$ thick sections were cut. The sections were stained with toluidine blue and were evaluated for demarcating up a representative area. Ultrathin sections were cut and the sections were evaluated with a transmission electron microscope (TEM model No. Morgagni 268D, Fei Company, 2002, Eindhoven, The Netherlands). In electron microscopic analysis, features of the individual myocyte at the ultrastructural level and collagen deposition in the interstitium were evaluated (magnifications $8000 \times$ and $5000 \times$ ). Representative light and electron 
micrographs of myocardium from the study group are depicted in Figures E2, E3, $A$ and $B$, and E4, $A$ and $B$.

\section{Definitions}

Cellular hypertrophy. Cellular hypertrophy was defined as an alteration of cardiac myocytes with an increase in cell size more than $15 \mu \mathrm{m}$ in diameter with significant nuclear enlargement. The hypertrophic changes were considered severe if the cardiac myocytes measured more than $20 \mu \mathrm{m}$ in diameter along with irregular cell shapes, lobulated nuclei, binucleate or multinucleate cells, enlarged T-tubules, abnormal Z-bands, increased amounts of sarcoplasmic reticulum, and multiple intercalated discs.

Myoelastofibrous endocardial thickening. Myoelastofibrous endocardial thickening was characterized by hyperplasia of elastic and smooth muscle with bundle formation. Elastic tissue hyperplasia, smooth muscle degeneration, and fibrous replacement are evolutionary phases of this lesion. The severity of endocardial thickening was graded as mild (1-2 mm), moderate $(2-5 \mathrm{~mm})$, and severe $(>5 \mathrm{~mm})$.

Myocyte degeneration. The degenerative changes observed in contractile elements of cardiac myocytes are known as myocytolysis. Myocytolysis of cyanotic patients with RVOT obstruction differs from the usual type of myocytolysis in that it is not associated with the formation of hypercontraction bands.

The degenerative changes were considered mild when there was focal loss of myofilaments, with proportionately greater loss of thick myosin filaments, thereby producing an apparent increase of the thin actin myofilaments. Additional early degenerative changes included focal thickening of Z-bands, fragmentation, and sarcolemmal accumulation of Z-band material.

The degenerative changes were considered severe in the presence of cellular atrophy, cellular and myofibrillar disorganization, myelin figure formation, myofibrillar lysis, proliferation of smooth endoplasmic reticulum, intrasarcoplasmic lipid deposition, abnormally small mitochondria, intramitochondrial deposits, loss of intercellular connections, and marked interstitial fibrosis.

Interstitial/perivascular/intermyocellular fibrosis. Fibrosis was defined as an increase in pericellular matrix with deposition of mature collagen bundles, microfibrils, and other ground substances, which in turn separate the histologically viable cardiac myocytes. Perivascular fibrosis differs from a replacement fibrosis in that the former is present in patients with RVOT obstruction and cellular hypertrophy.

Low cardiac output syndrome in repaired TOF. Low output syndrome was diagnosed if the patient required inotropic support (dopamine $\left[4-10 \mu \mathrm{g} \cdot \mathrm{kg}^{-1} \cdot \mathrm{min}^{-1}\right]$, dobutamine [5-10 $\left.\mu \mathrm{g} \cdot \mathrm{kg}^{-1} \cdot \min ^{-1}\right]$, epinephrine $\left[0.01-0.1 \mu \mathrm{g} \cdot \mathrm{kg}^{-1} \cdot \mathrm{min}^{-1}\right]$, milrinone $[50 \mu \mathrm{g} / \mathrm{kg}$ intravenous bolus followed by $0.375-0.75$ $\left.\mu \mathrm{g} \cdot \mathrm{kg}^{-1} \cdot \mathrm{min}^{-1}\right]$ ), either isolated or in combination, in the operating room or in the intensive care unit to maintain stable hemodynamics in the absence of residual structural lesions and mechanical external compression after correction of all electrolytes or blood gas abnormalities and after adjusting the preload to its optimal value. Low output syndrome was also diagnosed if there was an increasing requirement of the above-mentioned inotropes along with afterload reduction with sodium nitroprusside. Patients who received less than $4 \mu \mathrm{g} \cdot \mathrm{kg}^{-1} \cdot \mathrm{min}^{-1}$ of dopamine to increase renal perfusion were not considered to have low output syndrome.

Invasive monitors to measure cardiac output directly (SwanGanz catheter [Edwards LifeSciences, Irvine, Calif], pulmonary artery pressure line, and thermistors) are cumbersome and hazardous in children and are generally avoided in our setup except in complex cases. We generally limit intracardiac monitoring to right and left atrial pressure lines.

Accordingly, under the definition of low output syndrome after repair of TOF, an integration of relevant clinical, laboratory, and bedside echocardiographic criteria were used. The criteria for diagnosis were as follows: (1) cold extremities, absent pedal pulses, decreased toe temperature, reduced systolic pressure, impaired renal function and oliguria $\left(<1.0 \mathrm{~mL} \cdot \mathrm{kg}^{-1} \cdot \mathrm{h}^{-1}\right)$, metabolic acidosis, increased serum lactate levels $\geq 2.0 \mathrm{mmol} / \mathrm{L} \geq 2$ hours), low mixed venous oxygen saturation $(\leq 50 \%)$, and blunt sensorium; (2) after repair of TOF, if the mean right atrial pressure was 5 to $10 \mathrm{~mm}$ higher than mean left atrial pressure in the absence of right-to-left or left-to-right shunting, absence of an appreciable gradient in the right ventricle, RVOT, or pulmonary arterial outflow tract, and postrepair right ventricular/left ventricular peak systolic pressure ratio of 0.8 or less, an impairment of right ventricular function was suspected and confirmed by bedside echocardiography and/or cardiac catheterization.

\section{Statistical Analysis}

Data were analyzed with Biomedical Data Processing Statistical Software (University of California Press, Berkeley, Calif) and SPSS 10.0 statistical package (SPSS Inc, Chicago, Ill). Continuous and interval-related data were expressed as the mean \pm standard deviation. $\chi^{2}$ For linear trend was performed to identify the association of ventricular arrhythmia with the follow-up period. $\chi^{2}$ Tests were used to assess the association between independent and outcome variables (ie, cellular hypertrophy, endothelial thickening, and interstitial fibrosis). Univariate logistic regression was performed to calculate the relative risk and the $95 \%$ confidence interval (CI) for each independent variable.

A multivariate forward stepwise logistic regression model was used to identify independent risk factors for cellular hypertrophy, endocardial thickening, and interstitial fibrosis through the "enter" method. Selection of independent variables was a forward stepwise method with a critical probability value of .15 and .10 for variable inclusion and exclusion, respectively. Two-tailed probability was used in all of the statistical tests.

\section{Results}

There were 7 (3.8\%) perioperative deaths resulting from massive pulmonary bleeding $(\mathrm{n}=2)$, low output syndrome and multiorgan failure $(n=4)$, and sepsis $(n=1)$. All patients were routinely started on dopamine $\left(4 \mu \mathrm{g} \cdot \mathrm{kg}^{-1} \cdot \mathrm{min}^{-1}\right)$ to increase renal perfusion and sodium nitroprusside $(0.5$ $\left.\mu \mathrm{g} \cdot \mathrm{kg}^{-1} \cdot \min ^{-1}\right)$ to reduce afterload. Patients considered to have low output syndrome $(\mathrm{n}=56)$ required dopamine $(4-10$ $\left.\mu \mathrm{g} \cdot \mathrm{kg}^{-1} \cdot \min ^{-1}\right)$, dobutamine $\left(5-10 \mu \mathrm{g} \cdot \mathrm{kg}^{-1} \cdot \mathrm{min}^{-1}\right)$, epinephrine $\left(0.01-0.1 \mu \mathrm{g} \cdot \mathrm{kg}^{-1} \cdot \min ^{-1}\right)$, and milrinone $(50$ $\mu \mathrm{g} / \mathrm{kg}$ intravenous bolus followed by $0.375-0.75 \mu \mathrm{g} \cdot \mathrm{kg}^{-1}$. $\min ^{-1}$ ), either isolated or in combination. Twenty-seven 
$(81.8 \%)$ patients more than 15 years of age and $29(29.3 \%)$ patients between 4 and 15 years of age had predominant right ventricular dysfunction and low cardiac output $\left(\chi^{2}[1\right.$ degree of freedom $(d f)]=27.95 ; P<.001$; odds ratio $[\mathrm{OR}]=10.86 ; 95 \% \mathrm{CI}=3.75-33.10)$. These patients had evidence of restrictive right ventricular physiology. Median duration of inotropic requirement was 7 days (range, 7-10 days) in these patients. Patients with normal renal function were administered oral angiotensin-converting enzyme inhibitors before weaning from inotropic agents. Postoperatively, digoxin, diuretics, and angiotensin-converting enzyme inhibitors were weaned at varying time intervals. There were no late deaths.

\section{Follow-up}

Survivors $(n=176)$ underwent clinical examination, electrocardiography, and echocardiography every 3 months. The patients' clinical courses, need for cardiac medications, and late complications were monitored.

Follow-up was 100\% complete (range, 1 to 48 months) and yielded 392.5 patient-years of data with a mean follow-up time of 26.76 months ( $S D \pm 13.31$; range, 1-48 months). The actuarial survival at 48 months was $96.17 \% \pm 0.01 \%$. At their last follow-up, 170 (96.6\%) patients were in New York Heart Association functional classes I and II. Only 6 (3.4\%) patients were receiving diuretics and vasodilators late postoperatively and were in class III. Reoperation or reintervention was not required for any patient.

Left ventricular function was normal (ejection fraction $>$ $0.5)$ in 146 (79.8\%) patients and depressed in 37 (20.2\%) patients. Eighty-four (45.9\%) patients had mild and 61 (33.3\%) had moderate pulmonary regurgitation. Eight $(4.4 \%)$ patients had mild tricuspid regurgitation. There were no residual surgical problems in any patient.

Preoperative electrocardiographic studies demonstrated normal sinus rhythm $(\mathrm{n}=183)$, right ventricular hypertrophy $(\mathrm{n}=183)$, and single uniform ventricular extrasystoles (Lown grade $2, \mathrm{n}=24$ ). Postoperative studies demonstrated sinus rhythm $(\mathrm{n}=183)$, incomplete right bundle branch block $(\mathrm{n}=176)$, left bundle branch block $(\mathrm{n}=32)$, atrial flutter $(\mathrm{n}=12)$, and ventricular extrasystoles (Lown grade $2, \mathrm{n}=24)$. In all patients, ventricular arrhythmias noted preoperatively were still present at a follow-up of 6 to 12 months. There was no overlap of patients between the group with atrial flutter and the group with ventricular extrasystoles.

Ventricular arrhythmia of grade 2 was detected in 11 $(11.1 \%)$ patients in whom repair was performed between 4 and 15 years of age and in $13(39.4 \%)$ patients whose age at operation was 15 years or older. According to an additive logistic model, the effect of age at repair on the influence of ventricular arrhythmia was significant $\left(\chi^{2}\left[\begin{array}{ll}1 & d f\end{array}\right]=24.4\right.$; $P<.001 ;$ OR $=8.21 ; 95 \% \mathrm{CI}=2.96-23.11)$.
The relation of ventricular tachycardia to the surgical technique and to the interval between repair and electrocardiographic monitoring was also studied. Although the monitoring was performed over a short period of time, the duration of follow-up had a significant effect on the incidence of ventricular arrhythmia $\left(\chi_{\text {trend }}^{2}=4.223, P<.04\right)$. On follow-up echocardiogram, the right ventricular systolic pressure was less than $35 \mathrm{~mm} \mathrm{Hg}$ in all patients. There was no left-to-right shunt in any patient.

\section{Histopathology and Risk Factor Analysis}

Of 194 specimens, 11 were rejected for histopathologic analysis. These specimens exhibited morphologic artifacts resulting from inadequate fixation and were indistinguishable from those produced by acute hypoxia. The exclusion criteria for specimens that had inadequate fixation were cells with contraction bands, shortening of sarcomeres, nuclear distortion, intracellular edema, disruption of sacrolemmal and mitochondrial membranes, and dehiscence of intercalated discs. These artifacts were caused by contraction of the muscle during excision and fixation and are similar to those produced by acute hypoxia.

Fifty-one (27.9\%) myocardial specimens were found to be histologically normal. These specimens exhibited an orderly arrangement of bundles of cylindrical cardiac myocytes oriented with their longitudinal axes in parallel and connected end to end by intercalated discs. Cellular hypertrophy, myocytolysis with or without endocardial thickening, and interstitial fibrosis were present in 132 (72.1\%), 125 (68.3\%), 119 (65\%) specimens, respectively. Tables 1 and 2 summarize the OR for potential risk factors regarding cellular hypertrophy, endocardial thickening, and interstitial fibrosis. Analysis of each selected categorical variable is subsequently discussed.

\section{Cellular Hypertrophy}

A total of 132 (72.1\%) specimens had cellular hypertrophy (Figure E5). It was graded as moderate in $41(31.1 \%)$ and severe in $25(18.9 \%)$ specimens. These specimens exhibited significant cellular enlargement (diameter over $20 \mu \mathrm{m}, \mathrm{n}=$ 30) with nucleomegaly, irregular cell shapes, splitting Zbands, multiple intercalated discs, bizarre accumulation of Z-band material, and enlarged, dilated T-tubules. Assessed by univariate analysis, age more than 4 years, hematocrit more than $45 \%$, systemic arterial saturation less than $80 \%$, and RVEDP more than $12 \mathrm{~mm} \mathrm{Hg}$ were independent risk factors $(P<.05)$. By multivariate logistic regression, accounting for the effects of other factors, we identified 3 significant predictors for cellular hypertrophy (Tables 1 and 2).

Myocytolysis With or Without Endocardial Thickening Mycocytolysis was exhibited by $68.3 \%(\mathrm{n}=125)$ of myocardial specimens (Figures E2, E3, $A$ and $B$, and E5). It was 
TABLE 1. Odds ratio for potential clinical risk factors for cellular hypertrophy, myocytolysis, and endocardial thickening and interstitial fibrosis (univariate analysis)

\begin{tabular}{|c|c|c|c|c|c|c|c|c|c|c|}
\hline \multirow[b]{2}{*}{ Variables } & \multirow[b]{2}{*}{ No. } & \multicolumn{6}{|c|}{$\begin{array}{l}\text { Endocardial thickening } \\
\qquad(n=125)\end{array}$} & \multicolumn{3}{|c|}{ Interstitial fibrosis $(n=119$ ) } \\
\hline & & No. $(\%)$ & $\begin{array}{c}\text { Risk ratio } \\
\text { (95\% CI) }\end{array}$ & $\begin{array}{c}P \\
\text { value }\end{array}$ & No. $(\%)$ & $\begin{array}{c}\text { Risk ratio } \\
\text { (95\% CI) }\end{array}$ & $\begin{array}{c}P \\
\text { value }\end{array}$ & No. $(\%)$ & $\begin{array}{c}\text { Risk ratio } \\
\text { (95\% CI) }\end{array}$ & $\begin{array}{c}P \\
\text { value }\end{array}$ \\
\hline \multicolumn{11}{|l|}{ Age } \\
\hline$<4 \mathrm{y}$ & 51 & $30(58.8)$ & 2.30 & .02 & $27(52.9)$ & 2.562 & .006 & $27(52.9)$ & 0.422 & .012 \\
\hline$>4 \mathrm{y}$ & 132 & $102(77.3)$ & $(1.12-5.0)$ & & $98(74.2)$ & $(1.31-5.03)$ & & $92(69.7)$ & $(0.22-0.83)$ & \\
\hline \multicolumn{11}{|l|}{ Sex } \\
\hline Male & 76 & $49(64.5)$ & 1.96 & .053 & $43(56.6)$ & 2.517 & .005 & $38(50)$ & 3.818 & .000 \\
\hline Female & 107 & $83(77.6)$ & $(0.99-3.66)$ & & $82(76.6)$ & $(1.33-4.76)$ & & $81(75.7)$ & $(1.99-7.31)$ & \\
\hline \multicolumn{11}{|l|}{ Hematocrit } \\
\hline$<45 \%$ & 63 & $36(57.1)$ & 3.00 & .001 & $34(53.7)$ & 2.676 & .003 & $24(38.1)$ & 7.661 & .000 \\
\hline$>45 \%$ & 120 & $96(80)$ & $(1.53-5.86)$ & & $91(75.8)$ & $(1.40-5.12)$ & & $95(79.2)$ & (3.83-15.32) & \\
\hline \multicolumn{11}{|c|}{ Systemic arterial oxygen saturation } \\
\hline$<80 \%$ & 116 & $92(79.3)$ & 2.587 & .005 & $88(75.8)$ & 5.285 & .000 & $94(81)$ & 9.147 & .000 \\
\hline$>80 \%$ & 67 & $40(59.7)$ & $(1.33-5.02)$ & & $37(55.2)$ & $(2.69-10.39)$ & & $25(37.3)$ & $(4.52-18.52)$ & \\
\hline \multicolumn{11}{|c|}{ Right ventricular end-diastolic pressure } \\
\hline$<12 \mathrm{~mm} \mathrm{Hg}$ & 119 & $70(58.8)$ & 21.70 & .000 & $70(58.8)$ & 4.278 & .000 & $60(50.4)$ & 61.950 & .000 \\
\hline $12 \mathrm{~mm} \mathrm{Hg}$ & 64 & $62(96.8)$ & $(5.07-92.94)$ & & $55(85.9)$ & $(1.93-9.46)$ & & $59(92.1)$ & (8.32-461.37) & \\
\hline \multicolumn{11}{|c|}{ Previous Blalock-Taussig shunt } \\
\hline Yes & 59 & $47(79.6)$ & 1.8 & .16 & $41(69.5)$ & 1.8 & .94 & $39(66.1)$ & 1.07 & .96 \\
\hline No & 124 & $85(68.5)$ & $(0.81-4.05)$ & & 84 (67.7) & $(0.53-2.25)$ & & $80(64.5)$ & $(0.53-2.17)$ & \\
\hline
\end{tabular}

graded as moderate in $28(22.4 \%)$ and severe in $24(19.2 \%)$ specimens. The incidence of endocardial thickening of the cyanotic myocardium was higher in older age groups, patients with systemic desaturation (80\%), higher hematocrit $(>45 \%)$, and higher RVEDP (Tables 1 and 2 ).

\section{Interstitial Fibrosis}

Interstitial fibrosis was exhibited by $65 \%(\mathrm{n}=119)$ of myocardial specimens (Figures E4, $A$ and $B$, and E5). The degree of perivascular and interstitial fibrosis was graded as moderate in $11(9.2 \%)$ and severe in $9(7.6 \%)$ specimens. The commonest pattern of interstitial fibrosis was of interfiber type $(\mathrm{n}=74,62.2 \%)$. Perivascular fibrosis was present in $32(26.9 \%)$ specimens. Both perivascular and interfiber interstitial fibrosis were present in 13 (10.9\%) specimens.
Replacement fibrosis was not seen in any specimen. Interstitial fibrosis was only predicted by age greater than 4 years, systemic arterial desaturation, and higher RVEDP (Tables 1 and 2).

\section{Discussion}

Histopathologic studies of myocardium in patients with TOF have been limited because of the limited number of patients and restricted observations. ${ }^{6-9}$

\section{Myocardial Dysfunction in TOF}

Considerable interest has been generated concerning the "myocardial factor" in patients with cyanotic congenital heart disease, including TOF. ${ }^{3,6-9}$ Long-term studies of survivors of TOF repair have demonstrated a definite risk of

TABLE 2. Risk factors for cellular hypertrophy, endocardial thickening, and interstitial fibrosis in TOF by forward stepwise logistic regression analysis

\begin{tabular}{|c|c|c|c|c|c|c|}
\hline \multirow[b]{2}{*}{ Variables } & \multicolumn{2}{|c|}{ Cellular hypertrophy } & \multicolumn{2}{|c|}{ Endocardial thickening } & \multicolumn{2}{|c|}{ Interstial fibrosis } \\
\hline & Risk ratio $(95 \% \mathrm{CI})$ & $P$ value & Risk ratio $(95 \% \mathrm{CI})$ & $P$ value & Risk ratio $(95 \% \mathrm{CI})$ & $P$ value \\
\hline $\operatorname{Age}^{*}$ & $1.560(0.64-3.81)$ & .330 & $2.90(1.25-6.75)$ & .013 & $6.82(1.14-40.76)$ & .035 \\
\hline Sex* & $1.836(0.80-4.20)$ & .151 & $4.59(1.98-10.66)$ & .000 & $1.94(0.45-8.43)$ & .376 \\
\hline $\mathrm{Hct}^{*}$ & $2.183(0.95-5.02)$ & .066 & $2.93(1.28-6.73)$ & .011 & $12.70(2.56-62.96)$ & .002 \\
\hline $\mathrm{SaO}_{2}^{*}$ & $2.292(1.00-5.24)$ & .050 & $7.04(3.03-16.35)$ & .000 & $34.23(5.73-204.54)$ & .000 \\
\hline RVEDP* & 26.997 (5.19-140.52) & .000 & $6.09(2.31-16.03)$ & .000 & $451.44(12.30-16562.15)$ & .001 \\
\hline
\end{tabular}

TOF, Tetralogy of Fallot; $\mathrm{Cl}$, confidence interval; $\mathrm{Hct}$, hematocrit; $\mathrm{SaO}_{2}$, arterial oxygen saturation; RVEDP, right ventricular end-diastolic pressure.

*Variables with higher risk. 
late death, right ventricular failure, and arrhythmias, probably owing to myocardial dysfunction that is independent of the operative correction of the defect. ${ }^{1-3}$ Latent biventricular dysfunction and a decrease in left ventricular contractile reserve in repaired TOF has been described. ${ }^{1,3,10} \mathrm{Krym}-$ sky, ${ }^{11}$ in a histologic study of 55 patients with unrepaired TOF, found diffuse and focal cardiac sclerosis in young children and even infants. In our study, 35\% of patients had right ventricular dysfunction preoperatively and demonstrated a moderate-to-severe degree of myocyte degeneration and interstitial fibrosis compared with patients with preserved ventricular function (Tables 1 and 2). Logistic regression analysis accounting for the effects of other factors demonstrated the degree of preoperative hypoxemia (arterial oxygen saturation $<80 \%$ ), increased preoperative hematocrit $(>45 \%)$, age older than 4 years, and RVEDP greater than $12 \mathrm{~mm} \mathrm{Hg}$ as the potential "risk factors" for cardiomyocyte degeneration and interstitial fibrosis. Such changes could be the anatomic substrate for compliance or contractile abnormalities of the ventricle of patients with TOF.

Evaluation of Disease Severity and Analyses of Risk Factors for Development of Cellular Hypertrophy With or Without Myocyte Degeneration, Endocardial Thickening, and Interstitial Fibrosis

The status of the myocardium determines the long-term prognosis of patients with cyanotic congenital heart diseases. Degenerated myocytes do not contribute to contractile function of the heart. ${ }^{1-3,6-10}$

Myocardial hypertrophy was exhibited by $72 \%$ of myocardial specimens. These patients were older than 4 years, had higher hematocrit values, and elevated ventricular enddiastolic pressure. Myocardial hypertrophy implies increase in tissue mass without increase in the number of cells. This leads to myocardial ischemia, degeneration, and ultimately fibrosis. ${ }^{6-11}$ Fiber size variation and irregular cell shapes were found in $36.1 \%$ (66/183) of our total patients. Shearing forces are known to occur at side-to-side junctions owing to different rates or magnitudes of contraction of adjacent cells, which may be responsible for the asynchronous growth of cardiac myocytes and their irregular shapes. ${ }^{8,9}$

Our findings of binucleate or multinucleate cardiac myocytes might represent abortive attempts at cellular hyperplasia (ie, nuclear division without cellular division) or they could result from cellular fusion. We did not observe any nuclei with mitotic activity. Membrane system may also proliferate faster than or out of phase with other cell organelles. This was manifested by lobulated nuclei, enlarged, dilated T-tubules, increased amounts of sarcoplasmic reticulum, and multiple intercalated discs. Dilatation of Ttubules led to increase in cell surface area, which would allow greater contact between intracellular and extracellular environments, providing for greater transfer of nutrients, ions, and metabolites. ${ }^{6,7,9,10}$ As cardiomyocytes are a resting cell population, generalized cellular enlargement and nucleomegaly without concomitant cell division result in significant cellular hypertrophy seen at light microscopic evaluation.

We observed sarcoplasmic vacuolation and myofibrillar loss in $45.4 \%$ (30/66) of patients with cellular hypertrophy. Each patient with sarcoplasmic degenerative changes was an adult with elevated ventricular end-diastolic pressure and systemic arterial desaturation. Cytoplasmic areas adjacent to myelin figures contained variably distorted mitochondria and fat vacuoles. Intramitochondrial glycogen was more common in those patients who demonstrated systemic arterial desaturation. This change is thought to represent an effect of chronic hypoxia on the permeability of the outer mitochondrial membrane.${ }^{8,9}$ Cellular and myofiber disorganization were present in 119 patients. These were associated with one or more of the following: severe hypertrophy, age older than 15 years, elevated RVEDP $(>12 \mathrm{~mm} \mathrm{Hg}$ ), myocytolysis with moderate-to-severe endocardial thickening, and interstitial fibrosis. It appears that fibrosis produces separation of cells so that the normal tension exerted on a given cell by the contraction of adjacent cells is no longer present. As new sarcomeres form in areas of fibrosis, the abnormal mechanical forces may be oblique or perpendicular, rather than parallel to the longitudinal axis of the cells. Such forces, therefore, may result in irregular cellular organization and disarray of newly synthesized myofibrils. ${ }^{6-9}$ This finding is of clinical relevance as it can be a mechanism of myocardial dysfunction.

\section{Supraventricular and Ventricular Arrhythmias}

The incidence of arrhythmias necessitating treatment in patients after TOF repair is $2 \%$ to $4 \%$ for atrial fibrillation/ flutter, $3 \%$ to $4 \%$ for sustained ventricular tachycardia, and $2 \%$ to $4 \%$ for sudden cardiac death. ${ }^{1,3,4}$ A substantially increased QRS complex duration on the electrocardiogram may be a prognostic marker of ventricular tachycardia and sudden cardiac death, with a QRS duration greater than 180 ms sensitively predicting the occurrence of life-threatening ventricular ectopy. ${ }^{12}$ Holter monitoring detects a higher percentage of arrhythmias, with one recent report demonstrating that $19 \%$ of patients had sustained ventricular tachycardia, $42 \%$ of patients had nonsustained ventricular tachycardia, and $23 \%$ of patients had atrial fibrillation/ flutter on 24-hour monitoring. ${ }^{4}$ There have been no late cardiac deaths among the study group to date and most patients are symptom-free and without antiarrhythmic medications.

\section{What Are the Potential "Risk Factors" for Ventricular Arrhythmia in Patients With TOF, Even Before \\ Repair?}

We concur with the observations of others that the frequency of ventricular arrhythmia increases in older patients 
before repair of TOF. ${ }^{2-4}$ Necropsy studies have shown extensive myocardial fibrosis in adults with uncorrected TOF that is not detected in younger patients. ${ }^{8,13}$ In this study, $65 \%$ of the examined specimens demonstrated evidences of perivascular and interstitial fibrosis. Moderate and severe degrees of interstitial fibrosis were exhibited by $9.2 \%(\mathrm{n}=$ $11)$ and $7.6 \%(n=9)$ of myocardial specimens, respectively. Age more than 4 years, systemic arterial desaturation, and elevated RVEDP were the major predisposing risk factors for interstitial fibrosis.

Residual hemodynamic abnormalities after surgery (eg, right ventricular systolic and diastolic hypertension), increased right ventricular dimensions, and decreased right and left ventricular ejection fraction also predispose to ventricular arrhythmias. ${ }^{1,3,4,12,13}$ Generalized fibrosis may result in multiple macroreentry circuits. Additionally, the ventriculotomy may be the site of the macroreentry arrhythmias, and these may be particularly important in the presence of generalized fibrosis. ${ }^{4,13}$

In an attempt to preserve right ventricular mechanics, we were able to perform the operation either through a combined right atrial incision and, when necessary, an incision in the pulmonary artery, thus minimizing or eliminating a right ventriculotomy. This may also minimize substrate for ventricular arrhythmias arising from right ventricular incisions. This study has demonstrated that older age at repair had a significant influence on ventricular arrhythmias (39\% vs $7.3 \%$ for age $>15$ years vs $<15$ years, $P<.001$ ). Surgical technique did not affect the outcome of postoperative ventricular fibrillation $(P=.61)$.

\section{Comparison of Patients Less Than 2 Years Old With Those Over 15 Years Old}

Because late presentation of congenital heart disease is not unusual in the developing world, it is not uncommon to be faced with the grown-up patient with tetralogy physiology without previous palliation. They are more cyanotic and polycythemic and are indeed a different subset of Fallot's tetralogy from that encountered in the Western world. The youngest patient in this series undergoing intracardiac repair was 12 months of age. Despite an average age of just under 9 years in this study group, only about a third of the patients received shunts. Although our setting is a tertiary level center, the socioeconomic profile of the patients and the lack of health insurance benefit led to delayed referral and surgery, accounting for higher age of the patients.

The two groups of patients in this study population represent the extremes of age ( $<2$ years and $>15$ years) and of morphologic alterations. None of the patients in this series aged less than 2 years had cardiac failure or arrhythmias preoperatively. Virtually no degenerative changes were present in the myocardium of patients aged less than 2 years. However, these were present in patients over 15 years old (Tables 1 and 2). Twenty-seven (81.8\%) patients more than
15 years of age and $29(29.3 \%)$ patients between 4 and 15 years of age had predominant right ventricular dysfunction as a cause of low cardiac output $(P<.001)$. These patients had significant diastolic dysfunction of the right ventricle after intracardiac repair.

Significant diastolic dysfunction of the right ventricle after TOF repair may produce a "restrictive physiology" in which the stiff right ventricle demonstrates little true filling and behaves as an almost passive conduit for pulmonary blood flow. ${ }^{6,7,14}$ Our study has demonstrated the presence of moderate-to-severe degrees of endocardial thickening and perivascular fibrosis in all of these patients. The additional adverse effects of pulmonary regurgitation, the presence of a right ventriculotomy, and residual lesions such as a hemodynamically significant ventricular septal defect or RVOTO might be expected to influence the diastolic performance of the ventricle. However, the exact interrelation of these factors remains unknown.

Although it is impossible to relate directly a low cardiac output state to specific myocardial morphologic alterations; such a relation is suggested by the presence of disorganization and degeneration in the heart muscle of adult TOF. $2,3,6,7,13$ These findings in two groups of patients suggest that long-term cardiac myocyte hypertrophy results in structural abnormalities that are responsible for abnormal cardiac function.

\section{Limitations of the Study}

Because this is prospective study, follow-up is necessarily shorter than in previous retrospective studies. It could be argued that with time ventricular arrhythmia may develop in patients operated on at a younger age.

The issue regarding relations between left ventricular dysfunction and myocardial damage in TOF may require more direct evidence, and further studies combining radionuclide ventriculography, myocardial perfusion, and metabolic imaging are currently being undertaken.

Because of the variability of cell sizes in tissues from the same patients, and even within the same block of tissue, determination of the absolute relationship of cell size to myocardial hemodynamics would require quantitative cytometric analysis. It remains to be determined whether these pathologic changes are reversible.

\section{Clinical Implications and Recommendations}

The present study indicates that despite cellular hypertrophy observed with light microscopy, various components of cardiac myocytes do not enlarge or become numerous in a synchronous manner. The preponderance of degenerative change in older age groups, in patients with long-standing cyanosis and pressure overload, and in patients with clinically evident myocardial dysfunction and ventricular arrhythmias support the concept of early operative correction 
if technically appropriate. The presence of perivascular and interstitial fibrosis may be the substrate for electromechanical dissociation, thus predisposing them to ventricular electrical instability and diastolic dysfunction.

Advanced cases of TOF demonstrated sarcoplasmic degenerative changes and perivascular and interstitial fibrosis. Our observations highlight the importance of providing a maximal degree of intraoperative myocardial protection in older patients who are likely to have myocardial degeneration.

\section{References}

1. Cobanoglu A, Schultz JM. Total correction on tetralogy of Fallot in the first year of life: late results. Ann Thorac Surg. 2002;74:133-8.

2. John S, Kejriwal NK, Ravi Kumar E, Bashi VV, Mohanty BB, Sukumar IP. The clinical profile and surgical treatment of tetralogy of Fallot in adults: results of repair in 200 patients. Ann Thorac Surg. 1986;41: 502-6.

3. Geva T, Sandweiss BM, Gauvreau K, Lock JE, Powell AJ. Factors associated with impaired clinical status in long-term survivors of tetralogy of Fallot repair evaluated by magnetic resonance imaging. $J$ Am Coll Cardiol. 2004;43:1068-74.

4. Deanfield JE, McKenna WJ, Presbitero P, England D, Graham GR, Hallidie-Smith K. Ventricular arrhythmia in unrepaired and repaired tetralogy of Fallot. Br Heart J. 1984;52:77-81.

5. Cheitlin MD, Armstrong WF, Aurigemma GP, Beller GA, Bierman FZ, Davis JL, et al. Guideline update for the clinical application of echocardiography: summary article. J Am Soc Echocardiogr. 2003; 16:1091-110.

6. Egorova IF, Serov RA, Ngven'ia L, Plotnikova LR, Larin IA. Agerelated features of cardiomyocyte ultrastructure of the effluent region of the right ventricle in patients with Fallot's tetralogy. Arkh Patol. 1996;58:58-61.

7. Isomura T, Hisatomi K, Inuzuka H, Suzuki S, Hayashida N, Nishimi $\mathrm{M}$, et al. Ultrastructural alterations of right and left ventricular myocytes in tetralogy of Fallot. Kurume Med J. 1990;37:177-83.

8. Sakashita I, Matsukawa T, Ando T, Asano K. Morphological comparision of infundibular pulmonary stenosis and tetralogy of Fallot. Jpn Heart J. 1971;12:205-13.

9. Jones M, Ferrans VJ. Myocardial degeneration in congenital heart disease: comparision of morphologic findings in young and old patients with congenital heart disease associated with muscular obstruction to right ventricular outflow. Am J Cardiol. 1977;39:1051-63.

10. Ghai A, Silversides C, Harris L, Webb GD, Siu SC, Therrien J. Left ventricular dysfunction is a risk factor for sudden cardiac death in adults late after repair of tetralogy of Fallot. $\mathrm{J} \mathrm{Am} \mathrm{Coll} \mathrm{Cardiol.}$ 2002;40:1675-80.

11. Krymsky LD. Pathologic anatomy of congenital heart disease. Circulation. 1965;32:814-27.

12. Gatzoulis MA, Till JA, Somerville J, Redington AN. Mechanoelectrical interaction in tetralogy of Fallot: QRS prolongation relates to right ventricular size and predicts malignant ventricular arrhythmias and sudden death. Circulation. 1995;92:231-2.

13. Garson A, Nihill MR, McNamara DG, Cooley DA. Status of the adult and adolescent after repair of tetralogy of Fallot. Circulation. 1979; 59:1232-40

14. Gatzoulis MA, Clark AL, Cullen S. Right ventricular diastolic function 15 to 35 years after repair of tetralogy of Fallot. Circulation. 1995; 91:1775-81. 


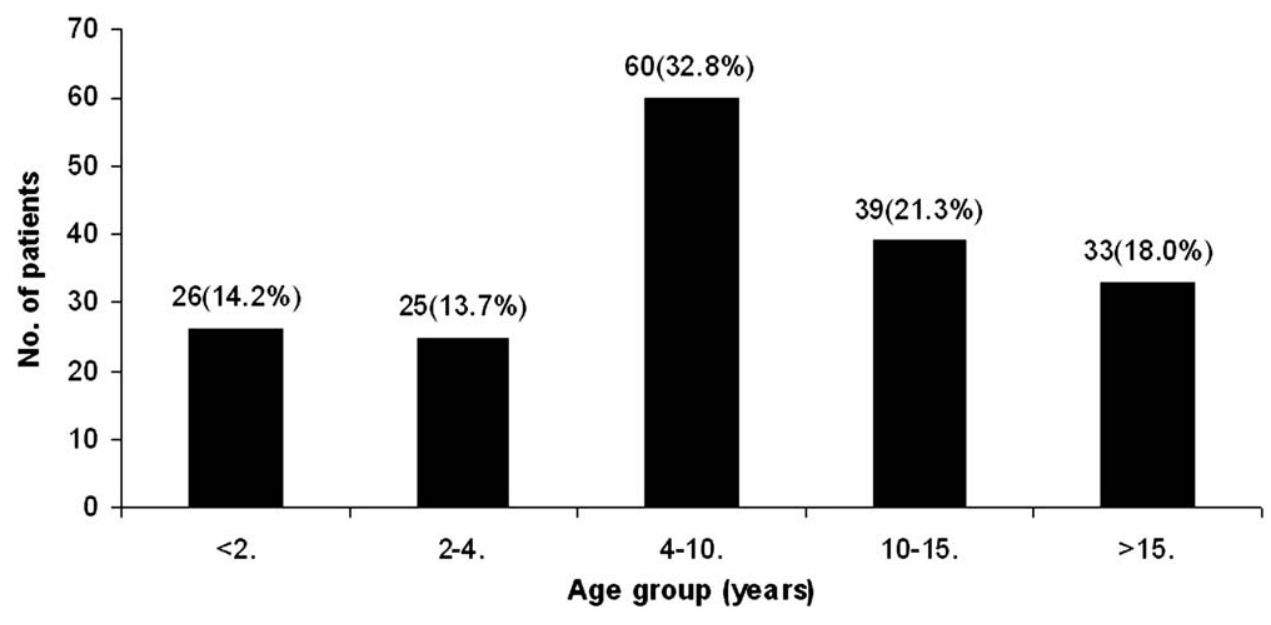

Figure E1. Age distribution of 183 patients undergoing intracardiac repair of TOF.

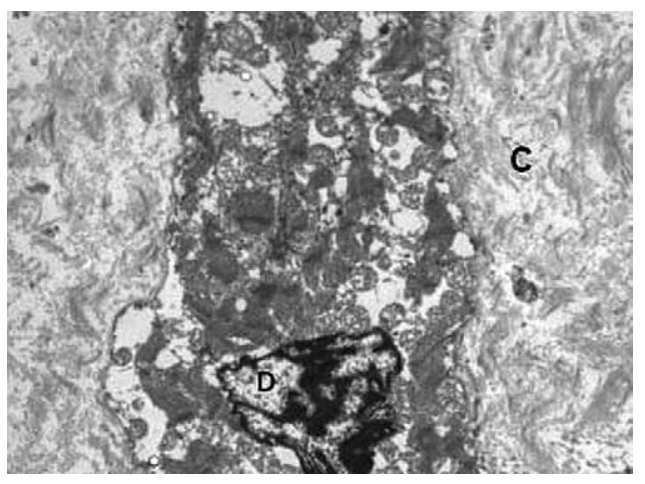

Figure E2. Transmission electron micrograph showing sarcoplasmic degenerative changes (D) within a myofiber with loss of contractile element. There is collagen deposition $(C)$ in the background. 

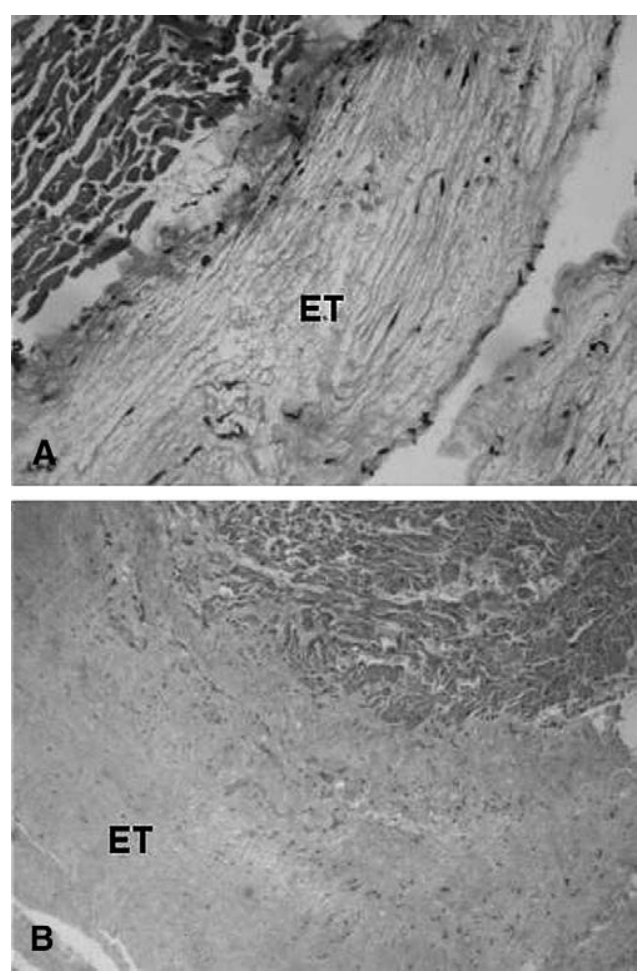

Figure E3. Photomicrograph from the myocardium showing (A) moderate endocardial thickening (ET) and (B) severe endocardial thickening (ET). 

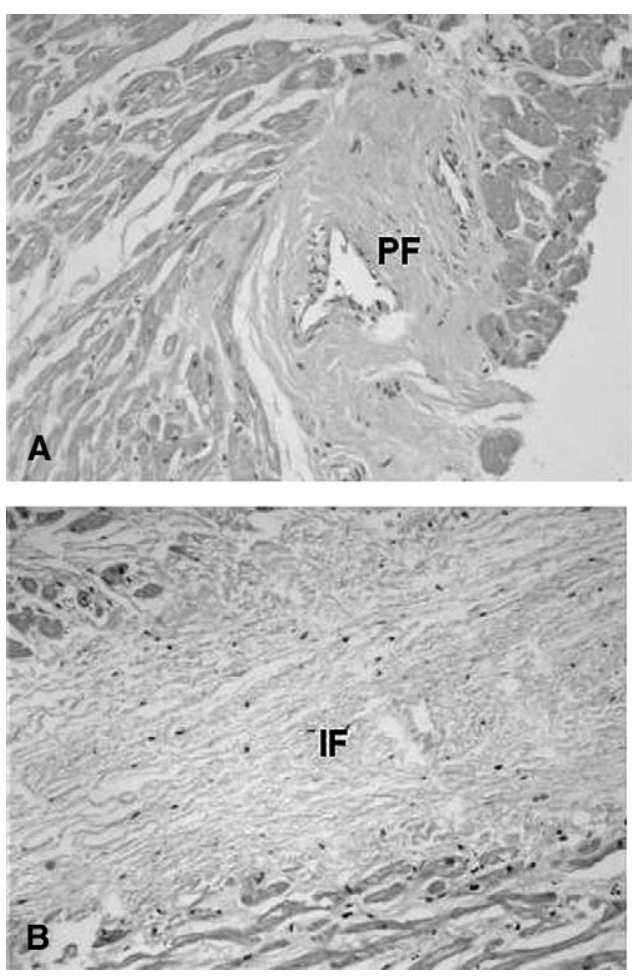

Figures E4. Photomicrograph from the myocardium depicting (A) perivascular fibrosis (PF) and (B) interstitial fibrosis (IF) (original magnification 180x).

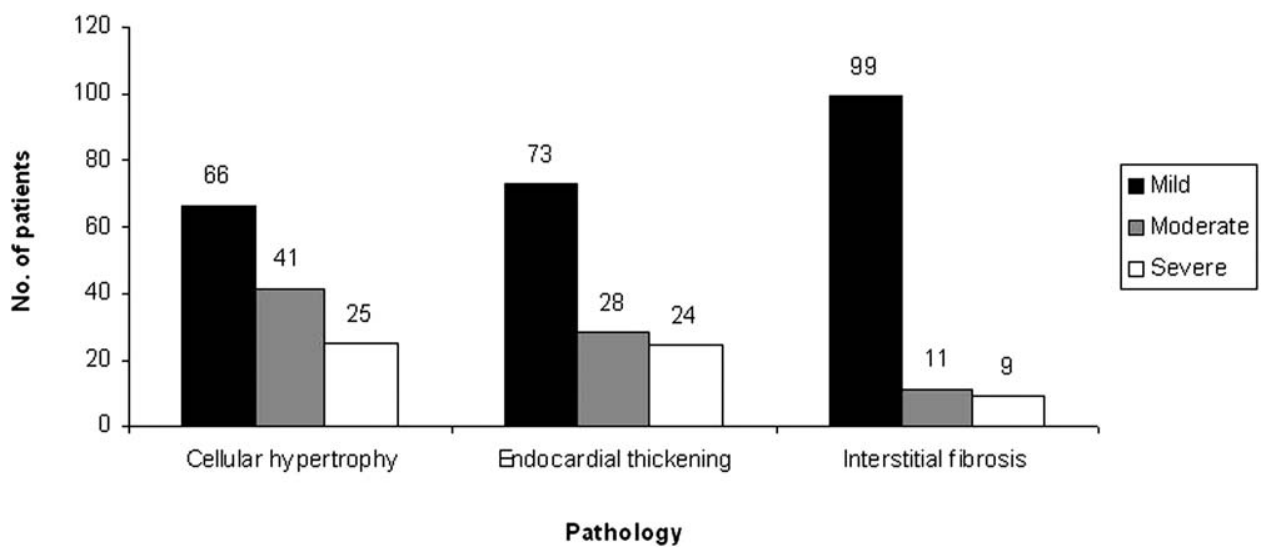

Figure E5. Histopathologic profile of the disease severity of resected muscles of RVOT of the study group (n=183). 
TABLE E1. Patient characteristics

\begin{tabular}{|c|c|}
\hline Patient-related variables & Mean \pm SD (range) \\
\hline Age at operation (mo) & $106.84 \pm 79.35$ (12 mo-42 y); median, 80 mo \\
\hline \multicolumn{2}{|r|}{ с } \\
\hline$<4 \mathrm{y}$ & 51 \\
\hline$>4 \mathrm{y}$ & 132 \\
\hline Male/female & $107: 76$ \\
\hline Body surface area $\left(\mathrm{m}^{2}\right)$ & $0.86 \pm 0.38\left(0.35-1.72 \mathrm{~m}^{2}\right)$ \\
\hline Previous Blalock-Taussig shunt & 59 \\
\hline Preoperative hemoglobin $(\mathrm{g} / \mathrm{dL})$ & $14.0 \pm 4.2(14-22)$ \\
\hline \multicolumn{2}{|l|}{ Preoperative hematocrit value } \\
\hline$<45 \%$ & 63 \\
\hline$>45 \%$ & 120 \\
\hline \multicolumn{2}{|l|}{ Preoperative right ventricular end-diastolic pressure } \\
\hline$<12 \mathrm{~mm} \mathrm{Hg}$ & 119 \\
\hline$>12 \mathrm{~mm} \mathrm{Hg}$ & 64 \\
\hline Preoperative systemic arterial oxygen saturation (\%) & $75.0 \pm 11.0(65 \%-84 \%)$ \\
\hline$<80 \%$ & 116 \\
\hline$>80 \%$ & 67 \\
\hline Coil embolization of major aortopulmonary collateral arteries & 37 \\
\hline Aortic crossclamp time (min) & $42.0 \pm 15.0$ \\
\hline Lowest temperature at operation $\left({ }^{\circ} \mathrm{C}\right)$ & $28^{\circ} \mathrm{C}$ \\
\hline Preoperative peak systolic pressure ratio & $0.96 \pm 0.03$ \\
\hline Postoperative peak systolic pressure ratio & $0.43 \pm 0.12$ \\
\hline Postoperative mean pulmonary artery pressure (mm Hg) & $15.0 \pm 3.0$ \\
\hline Coil embolization of major aortopulmonary collateral arteries & 37 \\
\hline
\end{tabular}

